\title{
Safety and Cost Implications of Same-Day Discharge Following Elective Percutaneous Closure of Patent Foramen Ovale and Atrial Septal Defects in Australia
}

\author{
Abhisheik Prashar ${ }^{1,2, *}$, Sanjana Shah ${ }^{1}$, Richard Zhang ${ }^{2}$, Kurt Mitchell ${ }^{1}$ and Mark Sader ${ }^{1,2}(\mathbb{D}$ \\ 1 Department of Cardiology, St. George Hospital, Gray Street, Kogarah, Sydney, NSW 2217, Australia; \\ sanjana.shah@health.nsw.gov.au (S.S.); kurt.mitchell@health.nsw.gov.au (K.M.); \\ mark.sader@health.nsw.gov.au (M.S.) \\ 2 Faculty of Medicine, University of New South Wales, Sydney, NSW 2052, Australia; \\ richard.zhang3@student.unsw.edu.au \\ * Correspondence: a.prashar@unsw.edu.au; Tel.: +61-02-91133172
}

\section{check for}

updates

Citation: Prashar, A.; Shah, S.; Zhang, R.; Mitchell, K.; Sader, M. Safety and Cost Implications of Same-Day Discharge Following Elective Percutaneous Closure of Patent Foramen Ovale and Atrial Septal Defects in Australia. Hearts 2021, 2, 543-550. https://doi.org/ $10.3390 /$ hearts 2040041

Academic Editor: Matthias Thielmann

Received: 6 October 2021

Accepted: 9 November 2021

Published: 9 November 2021

Publisher's Note: MDPI stays neutral with regard to jurisdictional claims in published maps and institutional affiliations.

Copyright: (c) 2021 by the authors. Licensee MDPI, Basel, Switzerland. This article is an open access article distributed under the terms and conditions of the Creative Commons Attribution (CC BY) license (https:/ / creativecommons.org/licenses/by/ $4.0 /)$.
Abstract: Background: Percutaneous closure of patent foramen ovale (PFO) and atrial septal defects (ASD) is being more commonly performed due to changes in international guidelines supporting its use. This study was performed to determine the clinical outcomes, safety and cost implications of same-day discharge (SDD) following such procedures and place this in an Australian context. Methods: This was a retrospective, observational study of patients undergoing elective percutaneous PFO or ASD closure at St. George Hospital, Australia between January 2011 and January 2020. Primary outcomes included 30-day major adverse cardiovascular endpoints (MACE) and readmission to hospital within 30 days. Results: Twenty-four patients were included in the primary analysis. Ten (41.7\%) patients underwent elective ASD closure while 14 (58.3\%) underwent PFO closure. Among the 24 patients who underwent elective percutaneous closure of structural heart disease, 23 patients $(95.8 \%)$ were managed with SDD. There were no MACE outcomes at 30 days. No patients were readmitted to hospital at 30 days following these procedures. When compared to overnight admission to hospital post-elective percutaneous structural heart condition closure, SDD yielded a cost saving of AUD 5999 per case. Conclusion: SDD following elective percutaneous closure of ASD and PFO was demonstrated to be a safe and effective strategy for managing patients. With more widespread use, it can lead to significant cost savings for hospitals without compromising patient care.

Keywords: patient discharge; foramen ovale; patent; heart septal defects; atrial; length of stay

\section{Introduction}

Patent foramen ovale (PFO) and atrial septal defects (ASD) remain two of the most common congenital cardiac anomalies. PFOs have a prevalence of up to $25 \%$ in adults, while ASDs have an incidence of around $0.1 \%$ in all live births [1,2]. Elective percutaneous closures of ASD and PFO are increasingly being performed in Australia following recent changes in international guidelines advocating the benefits of the procedure in selected individuals [3]. The current guidelines recommend percutaneous closure of PFOs where a temporal relationship between a PFO and a cryptogenic stroke can be established in patients under the age of 60 years. Percutaneous closure of secundum ASD is also indicated in the presence of hemodynamically significant shunts that cause platypnoea-orthodeoxia syndrome or right ventricular volume overload without pulmonary arterial hypertension or left ventricular disease, or indeed if there is clinical evidence of a paradoxical embolism likely arising from the ASD [4,5].

These procedures have historically been performed under trans-oesophageal echocardiography (TOE), guidance necessitating the use of general anaesthesia with prolonged procedural time [6,7]. Overnight admission to hospital has been the preferred, albeit uncommon, method of management specifically to monitor for peri-procedural complications. 
With the advent of fluoroscopy-guided closures and more recently, intracardiac echocardiography, same-day discharge (SDD) after these procedures has been made possible $[6,8]$. A meta-analysis conducted by Abaci et al. examining procedural complications of $\mathrm{PFO}$ and ASD closure in 28,142 patients across 203 studies reported that complication rates occurred in $1.4 \%$ of cases overall [9].

Many elective cardiology procedures can be safely managed as day-stay procedures including percutaneous coronary interventions, left atrial appendage closures and even transcatheter aortic valve replacements [10-12]. However, SDD after these procedures is more often proceduralist-driven than system-based. To the best of our knowledge, there are no large, randomised trial data or published consensus documents outlining the safety and feasibility of SDD after percutaneous PFO or ASD closure. Thus, the purpose of this retrospective, observational study was to evaluate the safety and cost-effectiveness of SDD following PFO and ASD closures in a single tertiary cardiology service in an Australian setting.

\section{Material and Methods}

\subsection{Patients}

This retrospective, observational study was conducted at St. George Hospital in Sydney, Australia between September 2011 and December 2020. St. George Hospital has been performing elective day-stay PFO and ASD closures since 2011. Consecutive patients who met the criteria for percutaneous PFO/ASD closure from September 2011 through to December 2020 were recruited as part of a registry. Their clinical, socio-demographic and procedural data was subsequently recorded into a database. Fluoroscopy data was collected using McKesson software v7.0 ${ }^{\mathrm{TM}}$.

\subsection{Procedure}

All patients fasted from midnight and were admitted on the morning of the procedure. PFOs were routinely closed under fluoroscopic guidance alone, whereas ASDs required TOE guidance. The need for general anaesthesia versus standard intravenous sedation with fentanyl or midazolam was assessed at the time of diagnostic TOE by the imaging cardiologist. Femoral venous access was gained using a 7-12 French femoral sheath. Intravenous heparin bolus was administered upon catheter passage into the left atrium. The femoral sheath was removed immediately after the procedure. All patients received a $6 \mathrm{~F}$ Abbott Perclose Proglide to achieve groin haemostasis. Procedural time was defined as the time from the administration of the local anaesthetic to removal of the catheter.

\subsection{Outcomes and Clinical Follow-Up}

Our primary outcomes included 30-day major adverse cardiovascular endpoints (MACE) and re-admission to hospital within the 30 days. Follow-up of all elective ASD and PFO closure patients was performed by Cardiology Departmental staff by reviewing the electronic medical records as well as contacting the patients by telephone. Patients or their next of kin were asked about complications following the procedure, including vascular access complications that may have required presentation to hospital or review by their local general practitioner. Data relating to mortality were also obtained from electronic hospital records and next of kin.

\subsection{Cost Determination}

The cost difference between overnight admission and SDD following elective ASD and PFO closure was calculated based on the information obtained from the finance department at our institution. We used national weighted activity unit calculators to gather specific cost estimates for overnight admission, post-procedure pathology costs and the cost for an overnight stay in a monitored bed in the coronary care unit. Readmissions to hospital were not included in the cost analysis. 


\section{Results}

During the period between September 2011 and December 2020, 24 patients underwent elective percutaneous PFO or ASD closure at our hospital. Table 1 outlines the baseline characteristics of these patients. Fourteen out of the twenty-four patients (58.3\%) underwent PFO closure, whereas the remaining ten $(41.7 \%)$ underwent ASD closure.

Table 1. Patient baseline characteristics.

\begin{tabular}{cc}
\hline Demographic & Total (N = 24) \\
\hline Age (mean \pm SD), years & $43.9 \pm 14.3$ \\
Male gender (n, \%) & $7(29.2)$ \\
BMI (mean \pm SD), kg/m $/ \mathrm{m}^{2}$ & $25.2 \pm 4.9$ \\
Smoking, current and past (n, \%) & $9(37.5)$ \\
Non-english-speaking background (n, \%) & $0(0)$ \\
Creatinine (mean \pm SD), umol/L & $70.8 \pm 22.9$ \\
Diabetes Mellitus (n, \%) & $4(16.7)$ \\
Hypercholesterolaemia, treated and untreated (n, \%) & $5(20.8)$ \\
Hypertension, treated and untreated (n, \%) & $5(20.8)$ \\
Atrial fibrillation (n, \%) & $3(12.5)$ \\
Previous TIA or CVA (n, \%) & $14(58.3)$ \\
Previous MI/PCI (n, \%) & $0(0)$ \\
\hline Values are N, mean \pm standard deviation. Abbreviations: BMI, body mass index; TIA, transient ischaemic attack; \\
CVA, cerebrovascular accident; MI, myocardial infarction; PCI, percutaneous coronary intervention.
\end{tabular}

The only indication for percutaneous ASD closure in our cohort was a secondum ASD defect with right ventricular dilatation. Cryptogenic cerebrovascular accident (CVA) was the most common indication for PFO closure in $78.6 \%$ of patients. Atrial septal aneurysm and recurrent CVA were the other indications in $57.1 \%$ and $21.5 \%$ of patients, respectively (Table 2). Most patients had more than one indication for the procedure, which have been considered separately.

Table 2. Indications.

\begin{tabular}{cc}
\hline Indication & Total (\%) \\
\hline ASD closure $(\mathrm{n}=10)$ & \\
Secondum ASD with RV dilatation & $10(100)$ \\
PFO closure ( $=14)$ & $11(78.6)$ \\
Cryptogenic CVA & $3(21.4)$ \\
Recurrent CVA & $8(57.1)$ \\
PFO with ASA & $3(21.4)$ \\
Migraine &
\end{tabular}

Abbreviations: ASD, atrial septal defect; RV, right ventricle; CVA, cerebrovascular accident; PFO, patent foramen ovale; ASA, atrial septal aneurysm.

Amongst the patients undergoing an ASD closure (occluder waist size range 22 to $38 \mathrm{~mm}$ ), nine patients received the AMPLATZER ${ }^{\mathrm{TM}}$ septal occluder (St. Jude Medical, St. Paul, MN, USA) and one received the OCCLUTECH ${ }^{\circledR}$ ASD occluder (Occlutech International $\mathrm{AB}$, Helsingborg, Sweden). Of the 14 patients undergoing a PFO closure, nine received the $25 \mathrm{~mm}$ AMPLATZER ${ }^{\mathrm{TM}}$ PFO occluder (St. Jude), three the $25 \mathrm{~mm}^{\text {OCCLUTECH }}{ }^{\circledR}$ PFO occluder (Occlutech International) and one the $30 \mathrm{~mm}$ AMPLATZER ${ }^{\mathrm{TM}}$ septal occluder [Table 3]. The mean duration of the procedure and fluoroscopy were $64 \pm 25 \mathrm{~min}$ and $7.6 \pm 6 \mathrm{~min}$, respectively. No procedural complications were observed.

Apart from one patient needing overnight admission due to staffing shortages in the cardiac catheterisation laboratory, the remainder of 23 patients $(95.8 \%)$ undergoing the percutaneous closure of $\mathrm{PFO} / \mathrm{ASD}$ were discharged on the same day. 
Table 3. Procedural characteristics.

\begin{tabular}{ccc}
\hline & Total & Percentage (\%) \\
\hline Implanted device (mm) & & \\
AMPLATZER $^{\mathrm{TM}}$ septal occluder & $10(1 / 1 / 4 / 1 / 1 / 1 / 1)$ & 41.7 \\
$(38 / 34 / 30 / 28 / 26 / 24 / 22)$ & $1(1)$ & 4.1 \\
Occlutech $^{\circledR}$ ASD occluder $(27)$ & $10(10)$ & 41.7 \\
AMPLATZER $^{\mathrm{TM}}$ PFO occluder $(25)$ & $3(2 / 1)$ & 12.5 \\
Occlutech $^{\circledR}$ PFO device $(30 / 25)$ & & 100 \\
Vascular access $^{\text {Femoral vein }}$ & 24 &
\end{tabular}

Abbreviations: ASD, atrial septal defect; PFO, patent foramen ovale.

All 24 patients (100\%) were successfully contacted over the phone as a part of routine follow-up after SDD. Six patients re-presented to the hospital within the first 30 days (Table 4). One patient was diagnosed with a transient ischaemic attack and was admitted to our hospital for two days. No infarct was seen on the magnetic resonance imaging scan of his brain. The other five were discharged from the emergency department with unrelated diagnoses in less than $24 \mathrm{~h}$.

Table 4. Clinical outcomes following SDD after elective ASD/PFO closure.

\begin{tabular}{cc}
\hline Outcomes at 30 Days & Total (\%) \\
\hline All re-hospitalisation & 6 \\
Unplanned cardiac re-hospitalisation & 0 \\
Urgent cardiac surgery & 0 \\
Major vascular complications & 0 \\
Myocardial infarction & 0 \\
Death & 0 \\
Stroke & 0 \\
\hline
\end{tabular}

The average cost for SDD and overnight admission after elective ASD/PFO closure at St. George Hospital are AUD 6558 and AUD 12,557, respectively. The cost of each device differed slightly and has been mentioned in Table 5 . The cost difference between SDD and overnight admission after elective ASD and PFO closures amounts to AUD 5999, which is the rate per night of a coronary care unit bed at our hospital. Patients that required several hours of monitoring beyond the standard $4-6 \mathrm{~h}$ of the procedure would be admitted as day-only admission, for which the charge was the same as overnight admission.

Table 5. Cost of each device used for ASD/PFO closure at St. George Hospital.

\begin{tabular}{cc}
\hline Device & Average Cost (AUD) \\
\hline AMPLATZER $^{\mathrm{TM}}$ septal occluder & 7436 \\
Occlutech $^{\circledR}$ ASD occluder & 5680 \\
AMPLATZER & 7436 \\
Occlutech $^{\circledR}$ PFO occluder & 5680 \\
\hline
\end{tabular}

\section{Discussion}

This single-centre, population-based study of patients presenting for elective percutaneous closure of PFO/ASD illustrates that the strategy of SDD is safe and cost-effective. Table 1 highlights the baseline characteristics of our study population. The mean age of our patient population was $43.9 \pm 14.3$ years. This roughly corresponds to the mean ages of 41.5-49.9 in recent trials [13-18]. The prevalence of traditional cardiovascular risk factors such as smoking $(37.5 \%)$, hypertension $(20.8 \%)$ and type 2 diabetes mellitus $(16.7 \%)$ in our cohort was also comparable to these studies, and reflective of the younger age profile of patients undergoing these procedures. 
Importantly, in our study there were no peri-procedural complications and no MACE outcomes, and all patients with the exception of one were discharged home after routine post-procedural monitoring for 4-6 h. No patient experienced any major or minor periprocedural complications over the course of our study, a rate significantly lower than the commonly reported complication rate of $1.4 \%$ [9]. Our data demonstrate a robust safety profile of SDD, with no unfavourable clinical outcomes related to the day-case procedure (Table 4).

In order to examine the safety of SDD, it is prudent to know the timing of all potential periprocedural complications, and whether any of these are addressable with an overnight stay compared to SDD. Most previous studies examining these complications provide very little insight into the timing of their onset. The CLOSE trial described new atrial fibrillation (AF) in 11 of 238 (4.6\%) patients undergoing PFO closure, with AF being detected on the same day of the procedure in three patients [14]. In our cohort, no patients experienced postprocedural AF. In a separate study, Amin et al. reported that of 11 recorded cases of cardiac erosions from PFO closure, 5 arose on the same day of the procedure, with none arising a day after [19]. In ASD closure, several studies report that complications arising within a day of the procedure include device embolization, cardiac erosions leading to pericardial effusion or tamponade, and new-onset AF [20-24]. Importantly, these studies make no mention of complications specifically following $24 \mathrm{~h}$ after the procedure. Taken together, these studies report no potential periprocedural complications manifesting at 24-48 $\mathrm{h}$ that would otherwise require prolonged observation and thus may justify overnight admission to hospital.

No randomised controlled trials have been conducted detailing the safety and efficacy of day-case percutaneous PFO or ASD closure. Evidence based on observational studies and case reports suggests that uncomplicated percutaneous PFO and ASD closure does not necessitate an overnight stay. Ponnuthurai et al. demonstrated that in uncomplicated PFO closure facilitated by intracardiac echocardiography, SDD resulted in no detrimental clinical or echocardiographic outcomes at follow-up 3 months after the procedure [6]. Barker et al. reported that of 467 patients undergoing percutaneous PFO closure, 456 had successful day-case surgeries, with low complication rates upon follow-up that were comparable to previous studies examining PFO closure complications [25]. While these studies have data showing that SDD is effective and feasible, this study is the first to examine the safety of SDD through analysis of 30-day MACE rates, a standard primary clinical endpoint for evaluating the safety of SDD after procedures involving catheterisation [10,26-29]. All patients were followed-up with no 30-day MACE outcomes.

To the best of our knowledge, no studies have analysed the potential cost savings of SDD compared to overnight admission in percutaneous PFO and ASD closure. As depicted in Table 5, SDD conferred a significant cost saving of AUD 5999 per procedure. This cost differential occurred primarily due to the absence of accommodation charges. In addition to being more cost-effective, it is also associated with greater patient satisfaction, as demonstrated by Chen et al. [30]. Adopting SDD as the standard approach to percutaneous PFO and ASD closure has the added benefit of eliminating cancellations due to bed unavailability. This has been a particular concern due to the changes in practice and service delivery for elective procedures brought about as a result of the COVID-19 pandemic. Implementation of SDD can eliminate barriers to these elective procedures. It is important to note that all procedures were performed by a single operator, eliminating inter-proceduralist variability that can influence complication rates and confound the safety of SDD. Other strengths of this study lie in the $100 \%$ follow-up rate, and in our analysis of cost savings from SDD, an oft-neglected but still significant endpoint.

\section{Limitations}

A limitation of our study was that the sample size was relatively small, and as such was underpowered to detect very rare periprocedural complications that may necessitate an overnight stay. Furthermore, as a retrospective cohort study, there remains the possibility 
of confounding and selection bias. Being a single-centre study, departmental protocols and resources may also limit the generalisability of our results. Sufficiently powered multi-centre randomised controlled trials should be conducted in order to best examine the safety and efficacy of SDD in percutaneous PFO and ASD closure. However, on the basis of our experience, SDD after uncomplicated percutaneous PFO or ASD closure remains a particularly attractive option that leads to safe, satisfying outcomes for patients and significant cost savings.

\section{Conclusions}

SDD is a favourable outcome after percutaneous $\mathrm{PFO}$ and ASD procedures, as demonstrated by our study. Day-case procedures are generally associated with decreased overall costs and increased patient satisfaction. While there is a lack of definitive guidance on SDD in the literature, there is some evidence that PFO and ASD closure could safely and effectively become day-case procedures. As such, a randomized controlled trial examining the safety and efficacy of SDD after PFO or ASD closure is merited.

Author Contributions: Conceptualization, A.P. and M.S.; methodology, A.P.; formal analysis, A.P. and S.S.; investigation A.P. and K.M.; resources, K.M.; data curation, K.M.; writing—original draft preparation, A.P., S.S. and R.Z.; writing—review and editing, S.S., A.P. and M.S.; supervision, A.P. and M.S.; project administration, S.S. and A.P. All authors have read and agreed to the published version of the manuscript.

Funding: This research received no external funding.

Institutional Review Board Statement: Not applicable.

Informed Consent Statement: Informed consent was obtained from all subjects involved in the study.

Conflicts of Interest: The authors have no financial or other interests in the products or distributors of the product or conflicts of interest to disclose.

$\begin{array}{ll}\text { Abbreviations } \\ \text { PFO } & \text { Patent foramen ovale } \\ \text { ASD } & \text { Atrial septal defects } \\ \text { TOE } & \text { Trans-oesophageal echocardiography } \\ \text { SDD } & \text { Same-day discharge } \\ \text { MACE } & \text { Major adverse cardiovascular endpoints } \\ \text { CVA } & \text { Cerebrovascular accidents } \\ \text { AF } & \text { Atrial fibrillation }\end{array}$

\section{References}

1. Homma, S.; Messé, S.R.; Rundek, T.; Sun, Y.P.; Franke, J.; Davidson, K.; Sievert, H.; Sacco, R.L.; Di Tullio, M.R. Patent foramen ovale. Nat. Rev. Dis. Primers 2016, 2, 15086. [CrossRef] [PubMed]

2. Geva, T.; Martins, J.D.; Wald, R.M. Atrial septal defects. Lancet 2014, 383, 1921-1932. [CrossRef]

3. Pristipino, C.; Sievert, H.; D’Ascenzo, F.; Louis Mas, J.; Meier, B.; Scacciatella, P.; Hildick-Smith, D.; Gaita, F.; Toni, D.; Kyrle, P.; et al. European position paper on the management of patients with patent foramen ovale. General approach and left circulation thromboembolism. Eur. Heart J. 2019, 40, 3182-3195. [CrossRef] [PubMed]

4. Baumgartner, H.; Bonhoeffer, P.; De Groot, N.M.; de Haan, F.; Deanfield, J.E.; Galie, N.; Gatzoulis, M.A.; Gohlke-Baerwolf, C.; Kaemmerer, H.; Kilner, P.; et al. ESC Guidelines for the management of grown-up congenital heart disease (new version 2010). Eur. Heart J. 2010, 31, 2915-2957. [CrossRef]

5. Stout, K.K.; Daniels, C.J.; Aboulhosn, J.A.; Bozkurt, B.; Broberg, C.S.; Colman, J.M.; Crumb, S.R.; Dearani, J.A.; Fuller, S.; Gurvitz, M.; et al. 2018 AHA/ACC Guideline for the Management of Adults with Congenital Heart Disease: A Report of the American College of Cardiology/American Heart Association Task Force on Clinical Practice Guidelines. J. Am. Coll. Cardiol. 2019, 73, e81-e192. [CrossRef]

6. Ponnuthurai, F.A.; van Gaal, W.J.; Burchell, A.; Mitchell, A.R.; Wilson, N.; Ormerod, O.J. Safety and feasibility of day case patent foramen ovale (PFO) closure facilitated by intracardiac echocardiography. Int. J. Cardiol. 2009, 131, 438-440. [CrossRef]

7. Meier, B. Closure of patent foramen ovale: Technique, pitfalls, complications, and follow up. Heart 2005, 91, 444-448. [CrossRef] 
8. Bijl, J.M.; Ruygrok, P.N.; Hornung, T.S.; Wilson, N.J.; West, T. Percutaneous closure of patent foramen ovale. Intern. Med. J. 2005, 35, 706-710. [CrossRef]

9. Abaci, A.; Unlu, S.; Alsancak, Y.; Kaya, U.; Sezenoz, B. Short and long term complications of device closure of atrial septal defect and patent foramen ovale: Meta-analysis of 28,142 patients from 203 studies. Cathet. Cardiovasc. Interv. 2013, 82, 1123-1138. [CrossRef]

10. Liew, S.; Dinh, D.; Liew, D.; Brennan, A.; Duffy, S.; Reid, C.; Lefkovits, J.; Stub, D. Prevalence, outcomes and cost implications of patients undergoing same day discharge after elective percutaneous coronary intervention in Australia. Heart Lung Circ. 2020, 29, e185-e193. [CrossRef]

11. Tan, B.E.; Boppana, L.K.; Abdullah, A.S.; Chuprun, D.; Shah, A.; Rao, M.; Bhatt, D.L.; Depta, J.P. Safety and Feasibility of Same-Day Discharge After Left Atrial Appendage Closure with the WATCHMAN Device. Circ. Cardiovasc. Interv. 2021, 131, 73-83.

12. Russo, M.J.; Okoh, A.K.; Stump, K.; Smith, M.; Erinne, I.; Johannesen, J.; Chaudhary, A.; Chiricolo, A.; Hakeem, A.; Lemaire, A.; et al. Safety and Feasibility of Same Day Discharge after Transcatheter Aortic Valve Replacement Post COVID-19. Struct. Heart 2021, 5, 182-185. [CrossRef]

13. Lee, P.H.; Song, J.-K.; Kim, J.S.; Heo, R.; Lee, S.; Kim, D.-H.; Song, J.-M.; Kang, D.-H.; Kwon, S.-U.; Kang, D.-W.; et al. Cryptogenic Stroke and High-Risk Patent Foramen Ovale: The DEFENSE-PFO Trial. J. Am. Coll. Cardiol. 2018, 71, 2335-2342. [CrossRef]

14. Mas, J.-L.; Derumeaux, G.; Guillon, B.; Massardier, E.; Hosseini, H.; Mechtouff, L.; Arquizan, C.; Béjot, Y.; Vuillier, F.; Detante, O.; et al. Patent Foramen Ovale Closure or Anticoagulation vs. Antiplatelets after Stroke. N. Engl. J. Med. 2017, 377, 1011-1021. [CrossRef]

15. Saver, J.L.; Carroll, J.D.; Thaler, D.E.; Smalling, R.W.; MacDonald, L.A.; Marks, D.S.; Tirschwell, D.L. Long-Term Outcomes of Patent Foramen Ovale Closure or Medical Therapy after Stroke. N. Engl. J. Med. 2017, 377, 1022-1032. [CrossRef]

16. Søndergaard, L.; Kasner, S.E.; Rhodes, J.F.; Andersen, G.; Iversen, H.K.; Nielsen-Kudsk, J.E.; Settergren, M.; Sjöstrand, C.; Roine, R.O.; Hildick-Smith, D.; et al. Patent Foramen Ovale Closure or Antiplatelet Therapy for Cryptogenic Stroke. N. Engl. J. Med. 2017, 377, 1033-1042. [CrossRef]

17. Altindag, T.; Roos-Hesselink, J.W.; Cuypers, J.A.A.E.; van Domburg, R.; de Jaegere, P.P.T.; Meijboom, F.J.; Witsenburg, M. Transcatheter device closure of atrial septal defects in patients aged 40 years and older. Neth. Heart J. 2010, 18, 537-542. [CrossRef]

18. Van De Bruaene, A.; Delcroix, M.; Pasquet, A.; De Backer, J.; Paelinck, B.; Morissens, M.; Budts, W. The importance of pulmonary artery pressures on late atrial arrhythmia in transcatheter and surgically closed ASD type secundum. Int. J. Cardiol. 2011, 152, 192-195. [CrossRef]

19. Amin, Z.; Hijazi, Z.M.; Bass, J.L.; Cheatham, J.P.; Hellenbrand, W.; Kleinman, C.S. PFO closure complications from the AGA registry. Cathet. Cardiovasc. Interv. 2008, 72, 74-79. [CrossRef]

20. DiBardino, D.J.; McElhinney, D.B.; Kaza, A.K.; Mayer, J.E., Jr. Analysis of the US Food and Drug Administration Manufacturer and User Facility Device Experience database for adverse events involving Amplatzer septal occluder devices and comparison with the Society of Thoracic Surgery congenital cardiac surgery database. J. Thorac. Cardiovasc. Surg. 2009, 137, $1334-1341$. [CrossRef]

21. Johnson, J.N.; Marquardt, M.L.; Ackerman, M.J.; Asirvatham, S.J.; Reeder, G.S.; Cabalka, A.K.; Cetta, F.; Hagler, D.J. Electrocardiographic changes and arrhythmias following percutaneous atrial septal defect and patent foramen ovale device closure. Catheter. Cardiovasc. Interv. 2011, 78, 254-261. [CrossRef]

22. Levi, D.S.; Moore, J.W. Embolization and retrieval of the Amplatzer septal occluder. Catheter. Cardiovasc. Interv. 2004, 61, 543-547. [CrossRef]

23. Nyboe, C.; Olsen, M.S.; Nielsen-Kudsk, J.E.; Hjortdal, V.E. Atrial fibrillation and stroke in adult patients with atrial septal defect and the long-term effect of closure. Heart 2015, 101, 706-711. [CrossRef]

24. Turner, D.R.; Owada, C.Y.; Sang, C.J., Jr.; Khan, M.; Lim, D.S. Closure of secundum atrial septal defects with the AMPLATZER septal occluder: A prospective, multicenter, post-approval study. Circ. Cardiovasc. Interv. 2017, 10, e004212. [CrossRef] [PubMed]

25. Barker, M.; Muthuppalaniappan, A.M.; Abrahamyan, L.; Osten, M.D.; Benson, L.N.; Bach, Y.; Ma, J.; Abraha, N.; Horlick, E. Periprocedural Outcomes of Fluoroscopy-Guided Patent Foramen Ovale Closure with Selective Use of Intracardiac Echocardiography. Can. J. Cardiol. 2020, 36, 1608-1615. [CrossRef]

26. Koutouzis, M.; Liontou, C.; Xenogiannis, I.; Tajti, P.; Tsiafoutis, I.; Lazaris, E.; Oikonomidis, N.; Kontopodis, E.; Rangan, B.; Brilakis, E. Same day discharge after chronic total occlusion interventions: A single center experience. Catheter. Cardiovasc. Interv. 2020, 1-8. [CrossRef]

27. Ranchord, A.M.; Prasad, S.; Seneviratne, S.K.; Simmonds, M.B.; Matsis, P.; Aitken, A.; A Harding, S. Same-day discharge is feasible and safe in the majority of elderly patients undergoing elective percutaneous coronary intervention. J. Invasive Cardiol. 2010, 22, 301-305. [PubMed]

28. Rodriguez-Araujo, G.; Cilingiroglu, M.; Mego, D.; Hakeem, A.; Lendel, V.; Cawich, I.; Paixao, A.; Marmagkiolis, K.; Flaherty, P.; Rollefson, W. Same versus next day discharge after elective transradial PCI: The RAdial SAme Day DischArge after PCI trial. (The RASADDA-PCI trial). Cardiovasc. Revasc. Med. 2018, 19, 7-11. [CrossRef] 
29. Shlofmitz, E.; Jeremias, A.; Goldberg, A.; Pappas, T.; Berke, A.; Petrossian, G.; Tsiamtsiouris, T.; Lituchy, A.; Lee, M.; Shlofmitz, R. Safety of Same-Day Discharge after Percutaneous Coronary Intervention with Orbital Atherectomy. Cardiovasc. Revasc. Med. 2019, 20, 573-576. [CrossRef] [PubMed]

30. Chen, Y.; Lin, F.F.; Marshall, A.P. Patient and family perceptions and experiences of same-day discharge following percutaneous coronary intervention and those kept overnight. Intensive Crit. Care Nurs. 2021, 62, 102947. [CrossRef] 